\title{
Particle morphology and magnetic properties of Ba0.5Sr0.5Fe12O19 powder calcined conventionally and by microwave heating
}

\begin{abstract}
Barium strontium hexaferrite (Ba0.5Sr0.5Fe12O19-BSF) has been prepared by solï gel process involving use of d-Fructose as a fuel. The prepared precursor was calcined in two different calcinations techniques conventional and microwave furnace. X-ray powder diffraction studies confirmed the formation of single phase Ba0.5Sr0.5Fe12O19. HR-SEM results show the morphology of the particles is hexagonal structures in platelet form. The average particle size of conventionally calcined BSF powder is $100 \mathrm{I} 250 \mathrm{~nm}$ and that of microwave calcined powder is $30 \mathrm{I} 100 \mathrm{~nm}$. The broad hysteresis loop reveals that powder is well crystallized and exhibits hard magnetic properties.
\end{abstract}

Keyword: Ceramics; Solï gel processes; Magnetization; Scanning electron microscopy, SEM 